\title{
Spreading dynamics of an impinging ferrofluid droplet on hydrophilic surfaces under uniform magnetic fields
}

\author{
Md Rifat Hassan* and Cheng Wang* \\ Department of Mechanical and Aerospace Engineering, Missouri University of Science and \\ Technology, 400 W. 13th St., Rolla, Missouri, 65409, USA \\ E-mail: mhvcp@mst.edu; wancheng@mst.edu
}

\section{Supporting Information Available}

\section{Validation of numerical model}

(a)

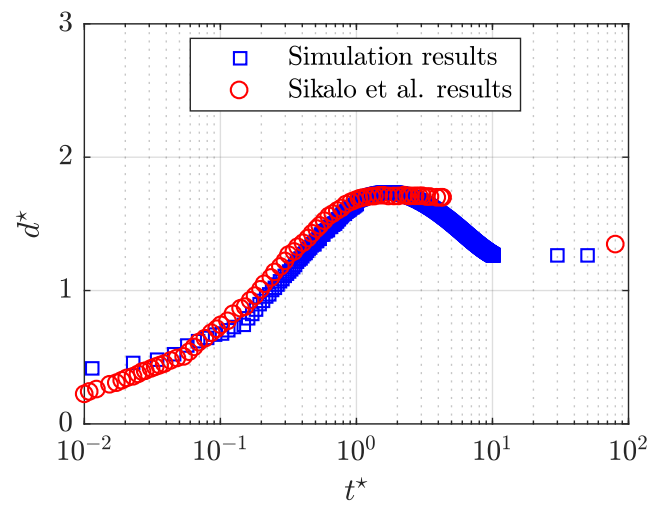

(b)

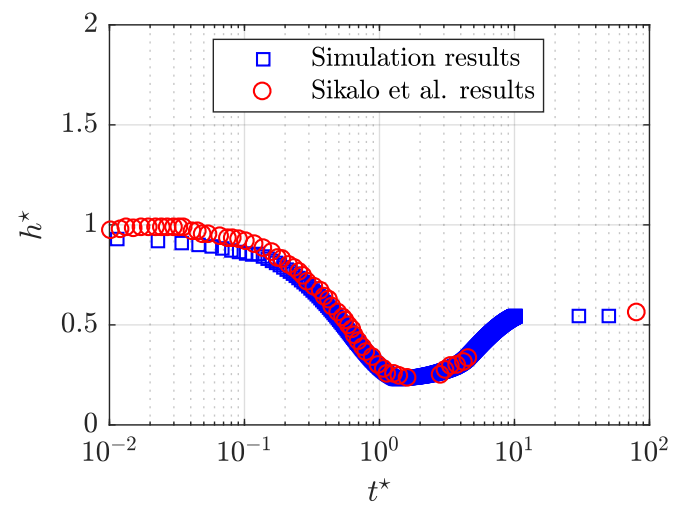

Figure S1: Comparison of spreading characteristics of a glycerin droplet on a solid surface at $\mathrm{Oh}=0.267, \mathrm{Re}=36$, and $\mathrm{We}=92$ against the experimental findings of Sikalo et al. ${ }^{1}$ (a) Spreading and recoiling of droplet, $d^{\star}$ vs. $t^{\star}$; and (b) Apex height of droplet, $h^{\star}$ vs. $t^{\star}$. 
In order to check the accuracy of the results, we also performed several validations of the numerical model against the existing experimental and theoretical findings in the literature. Figure S1 represents the spreading characteristics of a glycerin droplet on a solid surface under gravity at $\mathrm{Oh}=0.267, \mathrm{Re}=36$, and $\mathrm{We}=92$, and it can be observed that our simulation results (spreading and recoiling of droplet, apex height) quantitatively agree very well with the experimental findings of Sikalo et al. ${ }^{1}$ Note that, in this case, the simulations mimic the exact liquid properties and magnitude of different parameters that are used in the experiments performed by Sikalo et al. ${ }^{1}$

(a)

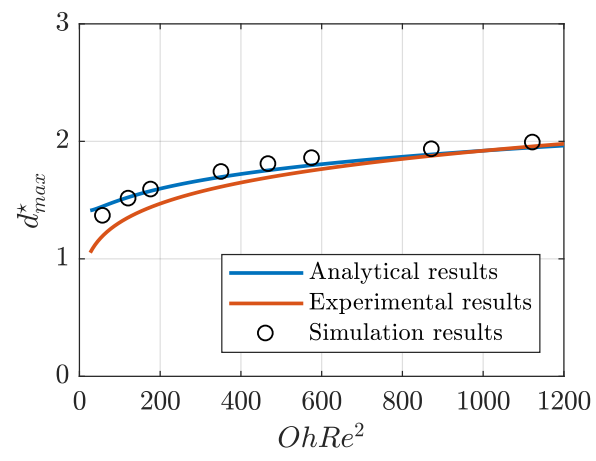

(b)

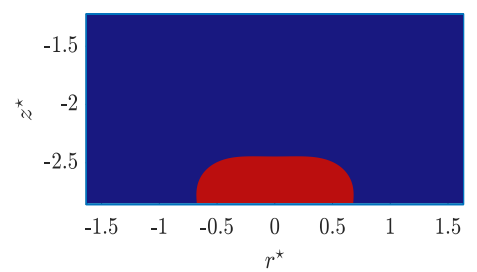

(c)

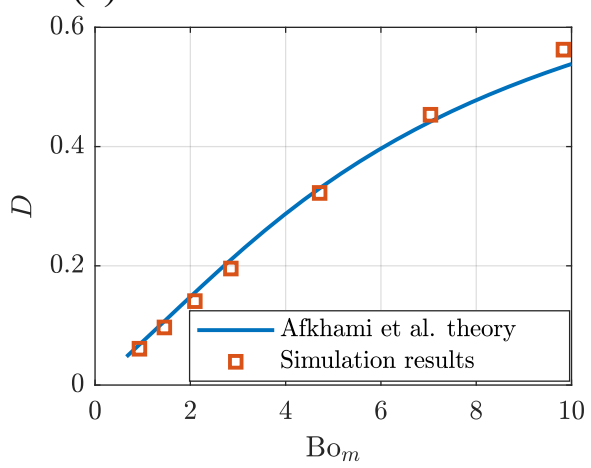

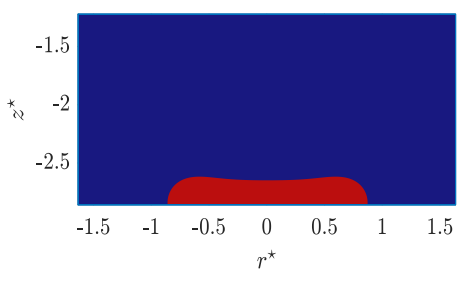

$O h R e^{2}=353$
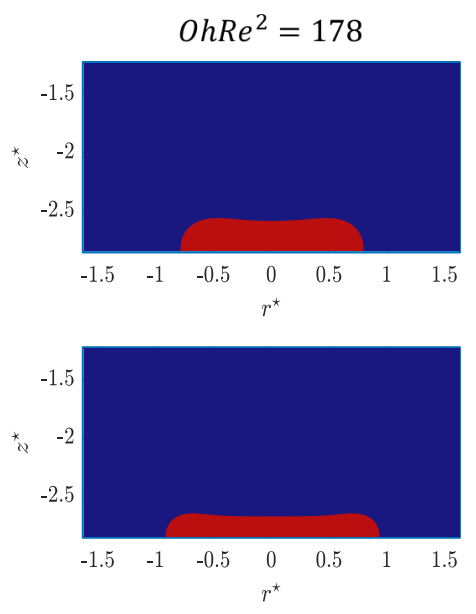

$\operatorname{OhRe}^{2}=577$

Figure S2: Comparison of simulated maximum spreading diameter $d_{\max }^{\star}$ of a Newtonian droplet against analytical ${ }^{2}$ (Chandra and Avedisian) and experimental ${ }^{3}$ (Scheller and Bousfield) findings in literature. (a) $d_{\max }^{\star}$ vs. $O h R e^{2}$; (b) Representative maximum droplet spreading diameter $d_{\text {max }}^{\star}$ under different $O h R e^{2}$; and (c) Deformation of a ferrofluid droplet in a quiescent flow under uniform magnetic fields, $D$ vs. $\mathrm{Bo}_{m}$.

Moreover, we compared the simulated maximum spreading diameter of the droplet during spreading against the prevalent theories and experiments in the literature. According to 
Chandra and Avedisian, ${ }^{2}$ for a given droplet diameter and impact velocity, the maximum spreading diameter $d_{\max }^{\star}$ of a Newtonian droplet can be theoretically approximated as:

$$
\frac{3}{2} \mathrm{ReOh}^{2} d_{\text {max }}^{\star}{ }^{4}+\left(1-\cos \theta_{w}\right) d_{\text {max }}^{\star}{ }^{2}-\left(\frac{\mathrm{Re}^{2} \mathrm{Oh}^{2}}{3}+4\right)=0
$$

whereas Schellar and Bousfield ${ }^{3}$ experimentally deduced an expression for the maximum spreading diameter $d_{\text {max }}^{\star}$ for a wide range of Reynolds and Ohnesorge numbers, which can be written as:

$$
d_{\max }^{\star}=0.61\left(\mathrm{Re}^{2} \mathrm{Oh}\right)^{0.166}
$$

Figure S2(a) represents the comparison of simulated maximum spreading diameter $d_{\max }^{\star}$ of a Newtonian droplet against the analytical (Chandra and Avedisian) and experimental (Scheller and Bousfield) findings as discussed above, and it clearly demonstrates a very good quantitative agreement among each other for a wide range of $\mathrm{OhRe}^{2}$. Furthermore, for better visualization purposes, a few representative two-dimensional (2D) shapes of maximum spreading droplet diameter are depicted in Figure S2(b). Additionally, the deformation of a ferrofluid droplet in a quiescent flow under uniform magnetic fields is depicted in Figure S2(c), which again agrees quantitatively well against the theory provided by Afkhami et al. ${ }^{4}$

\section{References}

(1) Šikalo, Š.; Marengo, M.; Tropea, C.; Ganić, E. Analysis of impact of droplets on horizontal surfaces. Experimental thermal and fluid science 2002, 25, 503-510.

(2) Chandra, S.; Avedisian, C. On the collision of a droplet with a solid surface. Proceedings of the Royal Society of London. Series A: Mathematical and Physical Sciences 1991, 432, 13-41.

(3) Scheller, B. L.; Bousfield, D. W. Newtonian drop impact with a solid surface. AIChE Journal 1995, 41, 1357-1367. 
(4) Afkhami, S. a.; Tyler, A.; Renardy, Y.; Renardy, M.; Pierre, T. S.; Woodward, R.; Riffle, J. S. Deformation of a hydrophobic ferrofluid droplet suspended in a viscous medium under uniform magnetic fields. Journal of Fluid Mechanics 2010, 663, 358-384. 